\title{
Certain Inverstigations on Privacy-Preserving Data Mining Using Three-Dimensional Spin Revolution (3DSR) Scheme
}

\author{
B. Santhosh $\operatorname{Kumar}^{1}$ (D) S. Karthik ${ }^{2} \cdot$ R. Cristin $^{1}$
}

Received: 22 August 2018/Accepted: 16 January 2021/Published online: 8 February 2021

(C) The Institution of Engineers (India) 2021

\begin{abstract}
The information disquiets are attractive in information extraction for safeguarding confidentiality. The key problem in information disquiets is to portray the equalization of two differing features as confidentiality safeguarding and information usage. The intention is to design a symmetrical information disquiets scheme by employing information disquiets and three-dimensional spins. Here the elements are segregated into clusters of three where each and every element is revolved about three pairs of alignments. The angle of revolution is chosen based on the difference in terms of confidentiality parameters which makes the actual reformation of information quiet intricate. Several information extraction schemes such as categorization and grouping are regular to symmetrical disquiets, and the information usage is conserved in the designed scheme. The results of the designed schemes offer improved confidentiality safeguarded outcomes and information usage against the conventional schemes.
\end{abstract}

B. Santhosh Kumar

santhosh.b@gmrit.edu.in

1 Department of Computer Science and Engineering, GMR Institute of Technology, Razam, Andhra Pradesh 532127, India

2 Department of Computer Science and Engineering, SNS College of Technology, Coimbatore, Tamilnadu 641035, India
Keywords Information extraction - Confidentiality · Scattered repository $\cdot$ Safeguarding $\cdot$ Spin revolution

\section{Introduction}

The intention is to design a symmetrical information disquiets scheme by employing information disquiets and three-dimensional spins. Here the elements are segregated into clusters of three where each and every element is revolved about three pairs of alignments. The angle of revolution is chosen based on the difference in terms of confidentiality parameters which makes the actual reformation of information quiet intricate. It is evident that the results of the designed schemes offer improved confidentiality safeguarded outcomes and information usage against the conventional schemes. Diverse information extraction scheme is prevailing which allows efficacious extraction of prototypes and data from immense volume of information. The firms employ these data to perform decision to acquire the approvals of the users. The information extraction offers efficacious improvements in domains such as machine-based learning, analysis and artificial intelligence which are commonly linked to information extraction which could possibly negotiate privacy.

The features aid escalating ethical disquiets related to the distribution of private data for information extraction actions. The confidentiality safeguarded information extraction (CSIE) scheme modifies the information to safeguard confidentiality. The CSIE is not only to safeguard the confidentiality during extraction stages, but it needs to regard the problems related to confidentiality in other stages of data exploration such as information operations and post-operations. It resolves the issues faced by the firm or an individual during the missing of delicate data 
or altered by the third-party information examiner. Therefore, the information required to be altered so that the thirdparty information examiner will not get pose any intention of the delicate data. In parallel, the usage of the information shall be conserved. The intention of the information disquiets is to announce combined data which could be employed for extraction without outflowing private data by presenting indecisions regarding sole values. It is estimated that the discerning multi-dimensional topographical data would aid in accomplishing improved safety along with the information effectiveness. Diverse information extractions such as sequential categorizers, support vector machine and Euclidean distance-based grouping schemes are different due to symmetrical discomposure. The aim is to focus a three-dimensional symmetrical spin of information designed to trouble the information before liberating them to the third-party information examiner.

The information extraction tools are focused to locate needful prototypes from an immense volume of information. These prototypes symbolize data and are represented in terms of choice-based hierarchies, groups and related policies. The data explored by diverse information extraction scheme might hold confidential data related to the individual or commerce. The safeguarding of confidentiality is a crucial feature of information extraction and the investigation of accomplishing some information extraction objectives without missing the confidentiality of the persons. The examination of confidentiality safeguarded information extraction schemes shall reflect the results of these schemes in extracting the outcomes along with confidentiality safeguarding. Within the restrictions of confidentiality, diverse schemes are designed for addressing the underlying issues. The accomplishment of confidentiality safeguarded information extraction scheme is calibrated in terms of their behaviour, information usage, indecision levels or conflicts of information extraction schemes. Therefore, no confidentiality safeguarding schemes are prevailing which overtakes all the prevailing conditions.

\section{Literature Survey}

\section{Information Extraction}

Rahman and Khan portrayed that the user relationship management system is employed to administer the associations among the firms with the prevailing and viewpoint users. The information extraction is employed in firms for performing choices and predicting the potential users [1]. Sachan et al. entailed that the information extraction schemes are employed widely for removing the hidden, formerly new and possibly needful data from the immense information set by making use of arithmetic and intellectual schemes [2]. Abdou et al. described that the information extraction is the mining of immensely attractive prototypes or data from an immense volume of information [3].

Noaman et al. described that the forecasting of nosocomial contaminations is a crucial segment of the hospital monitoring platform to permit the relevant individual to perform suitable defensive activities priory [4]. Ananthi Sheshasaayee and Logeshwari portrayed that the present day's advancement of trading promotion is enhanced based on the user classification designs [5]. Patel et al. portrayed that the information extraction is an interesting field due to its wide variety applications [6]. Therefore, there is no confidentiality security policy that overcomes all the prevailing conditions.

Antony Sheela and Vijayalakshmi entailed that the information extraction on perpendicularly or straight segmented information set has the overhead of safeguarding the confidential information [7]. Kumar et al. entailed that the information extraction schemes are increasing their importance for performing estimations location of unfamiliar prototypes to gain advantages from the user's information [8]. Sharma and Garg described that presently there prevails extreme force in distributing the private data and it creates problems in terms of data safety. When the information is mined from diverse sources or parties, a distress is created in terms of hiding which retards the information from distribution straightforwardly [9].

$\mathrm{Li}$ et al. described that the volume of internet-based information is crucially escalating because of the advancements of the networking schemes comprising the presence of big data [10]. Mohanrao and Karthik entailed that the process of information extraction is employed to locate the prototypes among the dozen of domains in the immense repositories. The prime dispute in information extraction is to preserve the confidentiality of private data [11]. Kaur and Kaur entailed that the information extraction is employed to mine crucial data from the voluminous information to hoard them and to review them in an efficient way. The concealed data could be mined from the immense set of information [12].

Yousef and Karunan describe that the remote sensors create an immense volume of information from satellites. Presently, there are immense needs for real-time information for remote sensing of applications and to mine needful data from the satellite images [13]. Liu and Chen offered illustrations on the mechanism of making use of information extraction schemes in locating patient chunks related to their favourites for required healthcare elements and their demographic features [14]. Saranya and Satheeskumar performed an analysis on medical image characteristic range using information extraction schemes. In the medical 
domain, there are diverse varieties of issues within the medical imaging such as categorization, separation, mining and mixture [15].

Lohiya et al. designed a removal of third-party prerequisites for preserving the privacy of the firm related information distributed among diverse firms at diverse extents in trade, promotions, hospital and entertainment areas [16]. Gupta and Bhadkamkar entailed that the primary intention of information extraction schemes is to attempt in the location of useful prototypes from the information which is immense in volume [17]. Mogtaba and Kambal portrayed that the confidentiality safeguarding is a great dispute in information extraction. The safety of the delicate data becomes a significant problem while discharging the information to the external parties [18]. Shinde et al. performed an assessment on the mechanism of how the supplier could assign the private information to the belief third parties in order that the outflow of information will be reduced by locating mortified mediators [19].

\section{Safeguarding}

Kelarev et al. performed an analysis on the present improved and conventional schemes for safeguarding the medical repositories [20]. Putri and Laksmiwati Hira entailed that presently the information is simply acquired universally and the issue of privacy or confidentiality of data becomes crucial since the data could be mined from the information by employing information extraction which occasionally might carelessly reveal these data [21].

Rajesh et al. entailed that the confidentiality safeguarding scheme has significant responsibilities to accomplish several information extraction processes on confidential information and to forward the information in a private manner to safeguard confidential information [22]. Verykios et al. designed generalizations of the fresh and quickly developing analysis of the confidentiality safeguarded information extraction. The design of categorization tree gathers the fundamental for examination [23].

Kumar and Santhi entailed that the requirement for information extraction along with the confidentiality safeguarding is grown as a need for swapping private data before disclosing the information over the network [24]. Cheng et al. entailed meanness comprising scarce and minimal score depicting excessive significance for safegaurding among the social networks, especially in the process like separation and identification [25]. Ge et al. described that the information extraction and assessment is playing a prominent part in data exploration and performing choices in the process firms over several decades. The computation-based engine in information extraction and assessment, machine learning assists as a fundamental tool for data mining, information framework identification and analysis [26].

\section{Confidentiality}

Gayathri and Poorna described that the escalated set, storage and assessment of individual precise information create severe disputes to safeguard the individualities to which the information belongs. In diverse circumstances, the mined data are extremely safe and require being refined before distribution for resolving the issues related to confidentiality [27]. Ramani et al. portrayed that the computation-based schemes are immensely helpful in medical image estimation to help the medical experts. Glaucoma is a vision menacing retinal disorder which requires care at the initial phases even though it does not disclose any indications [28].

Divecha and Mehta entailed that the preservation of privacy, confidential and safety analysis in information extraction is a major tendency. The prevailing improvements in the gathering information, information dispersal and correlated schemes has inducted a fresh age of analysis where the prevailing information extraction schemes shall be reviewed from the diverse viewpoints in terms of confidentiality safeguarding [29]. Lin et al. described that the confidentiality safeguarded information extraction is a growing issue which has become a crucial dispute in the past eras [30].

Asiabi and Tavoli entailed that the users are the most precious benefits of the firm. Because of their trade domain, it is mandatory to favour the user management of the firms [31]. Hasan et al. described that the bike distribution mechanism is environment-friendly systems which are extensive in smart cities. The intention is to analyse the issues related to confidentiality safeguarded bike distribution of microdata distribution [32].

\section{Privacy Used in Different Applications}

Alexandre et al. described that currently mobile slips are broadly employed for making purchases. Anyway, these applications have immense prospective in offering fresh services for the users like an exhibition of promotion operations based on the user favourites [33]. Rao and Kumar portrayed that the big data is created from several source-based communication, sensors and digital images, videos, audios and for diverse areas comprising healthcare termed as big data [34]. Dullaghan and Rozaki described that the telecommunication firms are immensely economic which portrays that the mobile suppliers require framework related to trade which could be employed in accomplishing a best possible extent of attention along with the minimal level of expenses in promotion-related actions [35]. 
Stankevicius and Kundeliene entailed that the prevailing categorization of the taxpayer is comparatively restricted and fixed. The current categorization-related issue linked to the taxpayer's performance needs current categorization examination schemes and frameworks which will examine the variation of the financial and psychographic representation of the taxpayers [36]. Farazzmanesh and Hosseini portrayed that in the present day's modest background the users are the most crucial benefit to any organization. Hence the firms shall comprehend the mechanism of maintenance and value of the Teamsters for each and every user [37].

Roshan and Afsharinezhad described that the information examination permits the firms to extract the prototypes and tendencies in their user's information for planning more efficient marketing suggestions followed by alterations within the marketing suggestions, assign promotion supplies effectively and enhance the user association management [38]. Chabanne et al. entailed that the neural networks are currently and extensively employed in machine-based learning where they are becoming deeper to precisely design or categorize the immense level of information constructs [39]. Pridmore and Hamalainen entailed that the promotions are always reliant on the input of fresh varieties of user's information through their past dependants on the conversions of this information into more and more efficient manner for directing and appealing the users [40].

Schutte et al. portrayed that the electronic banking is becoming very prevalent every day. The business colleges have recognized that the alteration to offer electronic-based services to their users for enduring appropriate and strive in an economic environment [41]. Abouelmehdi et al. described that the big data has basically altered the manner the firm preserves, organizes and escalates in any firms. A most talented domain where the big data could be used to make alterations is healthcare industry [42]. Aghdaie entailed that the providers are the crucial segment of dayto-day supply chain and firms more than they are dependent on their providers. Suitable victory in the present day's market is based on the manner they are efficacious firms dealing with the providers [43]. Panda et al. described that the progressive agreement search scheme is employed for its ability in locating solution space based on local and universal manner [44].

Rattanamethawong et al. performed an analysis to group the graduates into chunks for an improved recognition of their features, routines, a variety of performance and attention [45]. Bagul and Waghmare entailed that in the present day's modest firms among the globe is intended to preserve their users. The struggling firm requires creating their analytical prototypes to recognize the performance of their capable users [46]. Kade and Dhande portrayed that violence is growing every now and then and its existence is quite deep down in several parts of the globe. The immensely escalating violence actions make it very vital to govern these violations and halt its banquet violence through words, images and videos [47].

\section{Confidentiality Safeguarded Information Extraction (CSIE)}

The information extraction is a key process in data identification of repositories. The information extraction related analysis is focused on mining possibly helpful data from immense set of information for diverse application domains like managing user associations and estimation of marketplaces. The extracted data could be the prototypes, policies, groups or categorization prototypes. For the comprehensive process of information extraction, this information normally holds delicate and unique data like medicine- and finance-related data. The immense volume of information prevailing entails that it is probably to study immense data related to the persons from the public information. The confidentiality safeguarding is introduced as a crucial aspect in terms of attainment of the information extraction. The confidentiality safeguarded information extraction (CSIE) is focused on safeguarding the confidentiality of the unique information or delicate data without compromising the usage of the information. The individuals are more conscious about the confidentiality interferences on their private information and are very reluctant to distribute their delicate data. Presently the domains of the confidentiality are comprehended as the rapid improvements are due to the escalation in the capability to hoard the information. Precisely the present advancements in the domain of information extraction have paved way related to confidentiality. The intention of confidentiality safeguarded information extraction schemes extracts precise data from immense volume of information while safeguarding the identical time for considerable data.

The ultimate focus of the CSIE scheme is entailed below.

- The CSIE scheme shall have to retard the location of delicate data.

- It shall be resilient to diverse information extraction schemes.

- It shall not negotiate the access and the employment of non-delicate information.

- It shall not hold a fractional estimation-based difficulties.

Diverse safety-related standards are designed for information extraction and machine-based knowledge schemes for choice-based hierarchical categorization, grouping, and 
relationship policy-based extraction, neural networks and Bayesian networks. The key intention of these schemes is to safeguard the confidentiality of individualities delicate information which acquires needful data from the comprehensive information set. The analysed issues in information extraction are the process of exploring recurrent item sets and in parallel relationship policies. The relationship-based policy extractions are normally employed in diverse domains.

Diverse confidentiality safeguarded information extraction schemes make use of conversion which attempts in minimizing the utility of the prevailing information during its application on information extraction or routines. The confidentiality disquiets could retard the construction of supervised repositories dispersed among diverse places where no one is permitted to communicate their information to the other places. For confidentiality safeguarding of the information, the issue is related to the mechanism of acquiring the outcomes not based on the outcomes of extracted information. For illustrations, consider several hospitals' desire to acquire helpful and combined data related to a precise analysis based on the reports of the patients, while each and every hospital is not permitted because of their confidentiality actions to reveal the discrete confidential information. Hence they require being implemented on mutual and safe standards on their scattered repositories to attain their needed data.

For several cases, the information is scattered and appealing the information gathered in one place for estimation is not probable since these confidentiality actions or policies. Extraction of classification policies needs successive perusing of repositories which is quite expensive in operations. These schemes could be illustrated in supervising a scattered environment where the information could be scattered among diverse positions. The scattered repository conditions could be categorized as a straight segmented information and perpendicularly segmented information (Fig. 1).

\section{Parallel Segmentation of Information}

It segments the repositories into a number of non-overlying parallel segments. In these conditions, diverse places have diverse reports related to identical elements or individuals are employed for extraction. Diverse schemes make use of varieties of common schemes entailed for diverse issues.

\section{Perpendicularly Segmented Information}

For perpendicularly segmented information sets, each and every site has a diverse number of elements with an identical number of communication. The schemes of perpendicularly segmented extraction are protracted to the diverse difference of information extraction-based applications such as choice-based hierarchies, naïve Bayes categorizer and k-means grouping.

Fig. 1 Scattered repository

Centralized Data Mining

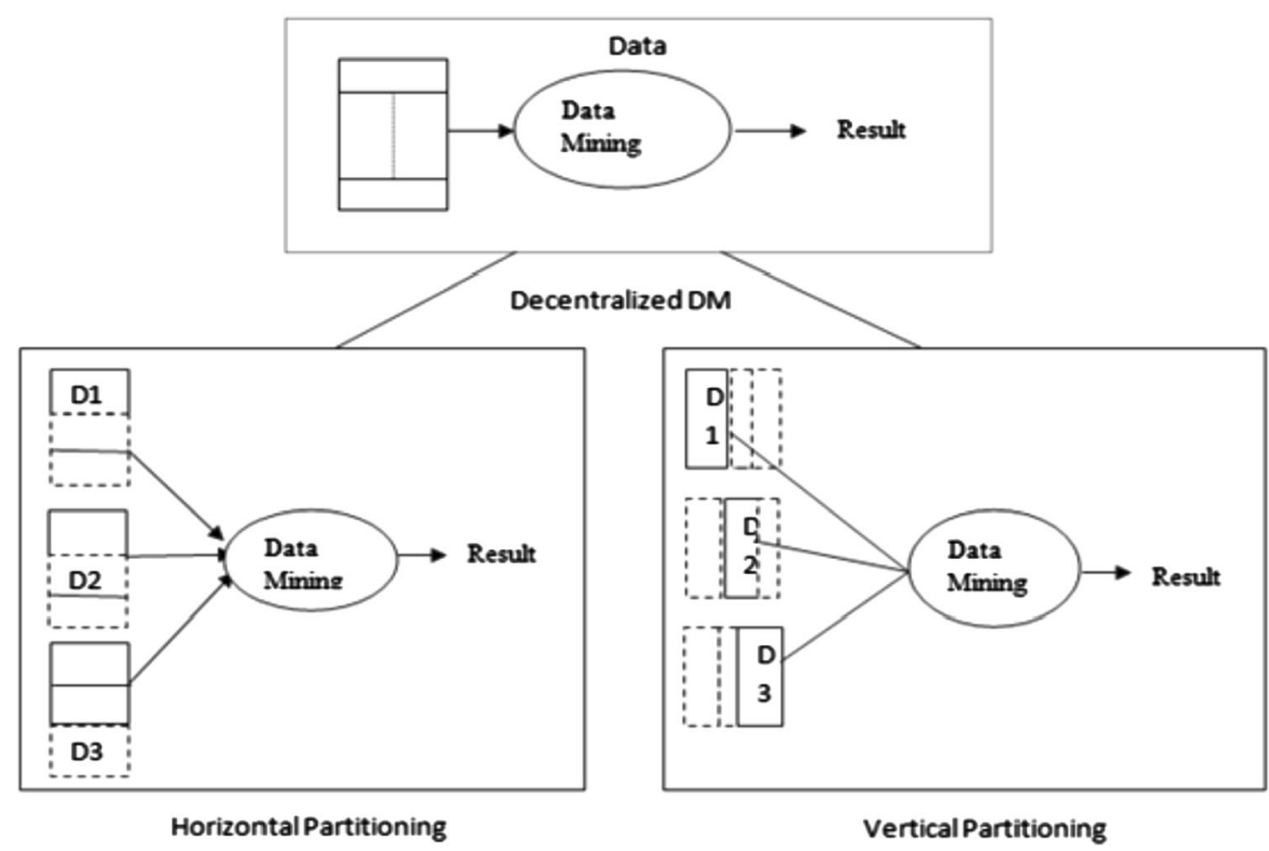




\section{Architecture of Confidentiality Safeguarded Information Extraction}

The architecture for CSIE is depicted in Fig. 2. For information extraction or data exploration from the repository, work on information collected by single/multiple firms and stored in their respective repositories. Then it is converted to a format appropriate for the process of examination stored in immense information repository, and the information extraction schemes are used for the creation of data/ instances. The initial level is unprocessed information or repositories during the presence of communications. The subsequent phase is the information extraction scheme and methods which assures confidentiality. The last phase is the outcomes of diverse information extraction schemes and techniques.

During phase 1, the unprocessed information gathered from solitary or diverse repositories or even information markets is converted into a layout which better suits for the determination of estimations. At this phase, the confidentiality-related disquiets are required to be considered. The analysis makes use of diverse schemes at this level to deal with processing of unprocessed information which is suitable for examination.

At Phase 2, the information from the information repository related to diverse operations which makes the information refines in order that it could be disclosed even to the untruthful information extractors. The operations used in this phase are jamming, dominance, alarm, alterations, customization and selection.

Followed by that, the information extraction schemes are used to the operating information for data/instance identification. Even the information extraction schemes are altered for the need of safeguarding confidentiality without compromising the intent of information extraction. At level 3 , the data/instances are disclosed by the information

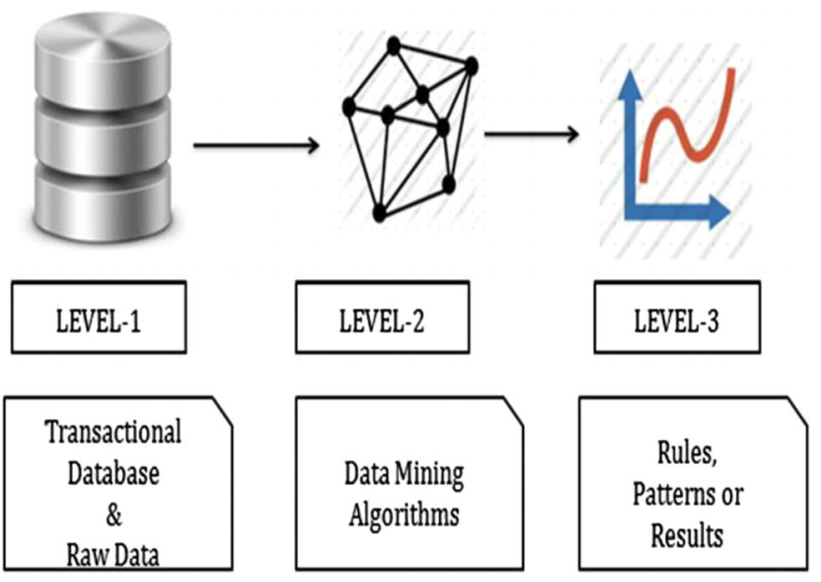

Fig. 2 CSIE architecture extraction schemes which are verified for its delicateness towards the threats in revealing.

\section{Confidentiality Safeguarded Information Extraction Schemes}

Presently, the observation is on the widespread analysis in the domain of CSIE. The analysis tracking on information extraction and arithmetic repositories the confidentiality safeguarded information extraction acquires considerable responsiveness and diverse scholars have conducted and improved volume of analysis in these domains. The confidentiality safeguarded information extraction has acquired escalated attention in information extraction, and the CSIE is becoming a crucial problem in examining the information extraction. As a result, the comprehensive fresh set of schemes are depicted to permit extraction of information meanwhile the time parting out the proclamation of any secret and delicate data.

The common of the prevailing schemes could be categorized into two widespread classes as

- Schemes safeguarding the delicate information within the extraction process.

- Schemes which safeguards the delicate information extraction created based on the applications of information extraction.

The initial category represents the scheme which is used for disquiets, specimen, sampling and modification schemes to the actual information sets for creating their refined complements which could be securely revealed to the untruthful parties. The intention is to classify the schemes to permit the information examiner to acquire precise information extraction outcomes in times of missing of real-time information.

Safe multi-party estimation schemes are designed to allow a number of information owners to mutually extract their information without having to disclose their information sets to one another. The subsequent classification is focused with the schemes which retard the revealing of delicate data prototypes extracted using the applications of information extraction schemes along the schemes for decreasing the efficiency of the classes within the process of categorization such that they do not disclose the delicate information. Differing from the supervised prototype, the scattered information extraction prototype receives the data relating to persons scattered across diverse places.

The schemes are designed within these domains for the issue of efficiently acquiring the outcomes of the extraction through these scattered sources. A straightforward scheme for information extraction over diverse sources will not distribute information to execute the prevailing 
information extraction tools at each and every place freely and aggregates the outcomes.

Therefore, it often miscarries to provide universally effective results. The problems create diversity among the local and universal outcomes comprising

- The values for a unique element might be segmented across the sources. The information extraction at the individual location will be incapable of identifying cross-site connections.

- At the same time, the item might be replicated at diverse locations and it will overbias the outcomes.

For a single position, it is probably to arrange identical populaces. The CSIE attempts to convert the actual information so that the outcomes of information extraction process shall not confront the restrictions of confidentiality. Based on the below-stated five features, the CSIE schemes could be categorized as

\section{Information (or) Policy Concealment}

This perception is related to whether the unprocessed information or the combined information shall be concealed. The information concealment refers to the safeguarding of delicate information values such as name, and identity number of people along with the policy concealment refers to safeguarding of private data within the data for instance relationship policies. The complexity for concealing the combined information in the form of policies is quite intricate, and for this tendency normally heuristics are designed.

\section{Information Delivery}

It entails information broadcasts. There are some schemes designed for the administered information, while other portrays a scattered information conditions. The scattered information conditions could be classified as parallel information segment and a perpendicular information segment. The perpendicular broadcast is entailed to the cases where different sets of records prevail in diverse places, while the perpendicular information broadcast relates that all the values for diverse elements prevailing in diverse places.

\section{Information Revisions}

The information revisions are employed with the intention of altering the exclusive values of a repository which desires to be permitted to the public and this manner assures elevated confidentiality safeguarding. The schemes of information revisions comprise
1. Discomposure

It permits the revision of element value by a fresh value (altering a 1 value to 0 or appending noise).

2. Hindering

It is the added prevailing element value with a question mark.

3. Exchange

It refers switching values of unique reports.

4. Specimen

It relates to missing the information for the only specimen of inhabitants.

5. Encoding

There are diverse cryptography based schemes used for encoding.

\section{Information Extraction Schemes}

For information extraction schemes, the confidentiality safeguarding scheme is proposed.

- Categorization-based information extraction scheme

- Relationship-based policy extraction scheme

- Grouping scheme

- Confidentiality safeguarding schemes

- Heuristic-based schemes

It is an adjustable alteration which changes only the chosen values which reduces the efficiency misses instead of all the prevailing values.

\section{Cryptography-Based Schemes}

The scheme comprises safe multi-party estimation for offering safe estimation during the conclusion of the estimation where no one is aware of anything excluding their individual input and the outcomes. The cryptography-based schemes are regarded for safeguarded confidentiality in scattered conditions by making use of encoding schemes.

\section{Renewal Based Schemes}

Here the actual scattering of the information is reconvened from the arbitrary information. Based on these measurement, diverse CSIE schemes might be categorized into the below entailed five classes (Table 1).

- Arbitrary CSIE,

- Disconnected CSIE,

- Arbitrarily reacted CSIE,

- Concentration scheme for CSIE,

- Cryptography-based CSIE. 
Table 1 Confidentiality safeguarded information extraction schemes

\begin{tabular}{|c|c|c|}
\hline Scheme & Merits & Restrictions \\
\hline Arbitrary CSIE & $\begin{array}{l}\text { Individuality or delicate information about the report possessors } \\
\text { must be concealed }\end{array}$ & $\begin{array}{l}\text { Association risks } \\
\text { Immense loss of data }\end{array}$ \\
\hline Disconnected CSIE & Here diverse elements are safeguarded autonomously & $\begin{array}{l}\text { Actual data values could not be recreated } \\
\text { Missing data }\end{array}$ \\
\hline Arbitrarily reacted CSIE & $\begin{array}{l}\text { It is quite effortless helping in concealment of data related to an } \\
\text { individual } \\
\text { Improved effectiveness of the cryptography-based CSIE }\end{array}$ & $\begin{array}{l}\text { Missing individual's data } \\
\text { It is not for diverse elements and repositories }\end{array}$ \\
\hline $\begin{array}{l}\text { Concentration } \\
\text { scheme for CSIE }\end{array}$ & $\begin{array}{l}\text { It makes use of false information instead of modified information } \\
\text { The scheme is a very real time in terms of linear information }\end{array}$ & $\begin{array}{l}\text { The immense volume of information is missed } \\
\text { It holds identical arrangements as the actual } \\
\text { information }\end{array}$ \\
\hline $\begin{array}{l}\text { Cryptography-based } \\
\text { CSIE }\end{array}$ & $\begin{array}{l}\text { The modified information is precise and must be safeguarded } \\
\text { Improved confidentiality as estimated against the arbitrary } \\
\text { schemes }\end{array}$ & $\begin{array}{l}\text { The scheme precisely is quite intricate to expand } \\
\text { diverse parties }\end{array}$ \\
\hline
\end{tabular}

\section{Assessment Standards of Confidentiality Safeguarded Scheme}

The confidentiality safeguarded information extracted is a crucial feature in the design and implementation of the schemes for finding appropriate assessment conditions and the design of connected policies. In some instances, there are no confidentiality safeguarding schemes prevailing which overcome the comprehensive schemes on all the probable measurements. Comparatively the scheme might accomplish improvements that other precise assessments such as behaviour and information usage. It is crucial to disseminate with a set of parameters which permits for choosing the best proper confidentiality safeguarded schemes for the information in terms of some precise metrics. The preliminary directives of assessment metrics are employed for assessing confidentiality safeguarded information extraction schemes as entailed below.

\section{a. Behaviour}

The behaviour of extraction scheme is calibrated in terms of time needed to accomplish the confidentiality conditions.

\section{b. Information Usage}

It is common calibration for information misses or misses within the features of information in offering the outcomes which could be created in the lack of CSIE schemes.

\section{c. Indecision Extents}

It is an evaluation of indecisions based on which the delicate data have to be concealed which still could be foreseen.

\section{d. Conflicts}

It is a degree of acceptance depicted based on the CSIE scheme against diverse information extraction schemes and prototypes. The entire aforementioned are analysed above the demand to be measured for improved assessment of confidentiality safeguarding schemes, but still two very crucial schemes are measuring confidentiality and missing data. The measurement of confidentiality or its parameters represents how carefully the actual value of an element could be measured. In case if it is evaluated with increased assurances, the confidentiality is minimal and vice versa. The missing accuracy in analysing the actual information set is termed as missing data which could be a reason for the disappointment of the intention of information extraction. This requires an equalization to be accomplished with confidentiality and missing data.

\section{e. Genesis}

Conventionally diverse schemes are designed by several researches and clusters to safeguard confidentiality in information extraction; preliminarily, several schemes such as arbitrary aggregation and reproduction are established that were subjected to nearly all sorts of risks. A lately diverse effective scheme which preserves the equalization among the information utility and confidentiality is designed. Diverse key schemes are such as information disquiets, information exchange, k-concealments, cryptographic schemes, policy concealment schemes and safely scattered extraction schemes.

There are two key information disquiets schemes, namely possibility scattering scheme and information value alteration scheme. For possibility scattering schemes, the information is swapped with other section from the same 
broadcasts. In information value alteration, the information segments are disturbed by either augmented noise, replicative noise or other forms of arbitrary schemes. The noise augmented disquiets disturbs the information set based on the aggregation of noise. Most commonly, the Gaussian-based scattering is employed in creating the noise values. More the association of noise it is identical to the actual information, the more the confidentiality is safeguarded. The initial module-based examination and the Bayes evaluation schemes are examined broadly for rebuilding the dislikes of arbitrary schemes. Additional schemes of disquiets comprise duplicative disquiets, spin disquiets and multi-dimensional disquiet. Other schemes like log-based modification are used to the information initially followed by which a pre-planned multivariate Gaussian-based noise is appended and lastly antilog of the noise appended information.

For information exchange the repository set is modified by exchanging values of the delicate elements among the files and there creating indecision about the delicate information. A k-secrecy prototype makes use of information overview and dominance components where the information is unconfined only if the data for each and every person held in the discharge could not be differentiated from at least $(k-1)$ other individuals. In the $k$ hierarchy-based disquiets, the information is segmented repeatedly into miniature subsets and the delicate information within the subsets are disconnected employing the subset average. Confidentiality safeguarded and scattered information extraction schemes are based on the duplicative arbitrary forecast matrices designed to safeguard the analytical features of information while enhancing the extents of confidentiality. The design of multi-party cooperation on confidentiality safeguarded extraction schemes safety combining a variety of symmetrical concerns is chosen on the basis of the various parties by making use of the keys. Along with the linked policy concealment scheme, the repository is managed to conceal the delicate policies. Fresh information extraction schemes such as arbitrary decision hierarchies, altered Bayesian networks and SVM categoriser are designed based on the CSIE schemes.

The goal is to extend the analysis performed for twodimensional spins which are employed as a scheme for information alteration for safeguarding the confidentiality. Within the designed scheme, the elements are segmented into three clusters and then spin disquiets are used in way that the information safeguards the internal Euclidian distances.

\section{Methodologies}

\section{Min_Max Standardization}

The standardization scheme employed in MIN_MAX schemes which match the value of an element ' $n$ ' prevails among the extent min and max value ' $n_{0}$ ' which prevails among the extent freshmin and freshmax.

$n_{0}=\frac{(n-\min )}{\max -\min } *($ fresh $\max -$ fresh $\min )+$ fresh $\min$

Therefore, for normalizing the information all the values of the elements are matched among the extents 0.0-5.0.

\section{Three-Dimensional Spins}

For the two-dimensional spin, the alignment of spin is always vertical to the $x y$ plane, i.e. the $z$-axis. For threedimensional spin, the alignment of spin could have any coordination in the space, i.e. the $x$-axis or $y$-axis or the $z$ axis based on the fundamental plane. The spin matrix, equations and the illustrations on space for each and every alignments of spin are depicted in Table 2.

Within the two-dimensional spins, the information is revolved along diverse alignments for improved information disquiets, i.e. the three pair alignments $x y, y z$ and $x z$. The employment of connective nature of the matrix the spin matrices $S_{x y}, S_{y z}$ and $S_{x z}$ could be measured as in Table 2.

\section{Proposed Scheme}

The intention is to design a three-dimensional spin-based alteration scheme which changes the information based on revolving three elements at a time along with two dissimilar alignments without negotiating the outcomes of extractions.

\section{Pre-Planning}

The information matrix $i_{\mathrm{m}}$ is considered to have only number elements. The information matrix before the disquiets required to be standardized to normalize them in order that during spins the Euclidian distance among the points persists the same. The standardization scheme employed is the MIN_MAX scheme.

$S_{x y}=S_{x} * S_{y}=\left(\begin{array}{ccc}\cos \theta & 0 & -\sin \theta \\ \sin ^{2} \theta & \cos \theta & \sin \theta \cos \theta \\ \sin \theta \cos \theta & -\sin \theta & \cos ^{2} \theta\end{array}\right)$ 
Table 2 Three-dimensional spins

\begin{tabular}{|c|c|c|c|c|}
\hline Alignment of spins & Equations & Spin matrix & & \\
\hline$Z$ alignment spin & $\begin{array}{l}x^{\prime}=x * \cos \theta-y * \sin \theta \\
y^{\prime}=x * \sin \theta+y * \cos \theta \\
z^{\prime}=z\end{array}$ & $S_{z}(\theta)=$ & $\left(\begin{array}{c}\cos \theta \\
\sin \theta \\
0\end{array}\right.$ & $\left.\begin{array}{cc}-\sin \theta & 0 \\
\cos \theta & 0 \\
0 & 1\end{array}\right)$ \\
\hline $\begin{array}{l}X \text { alignment spin } \\
Y \text { alignment spin }\end{array}$ & $\begin{array}{l}y^{\prime}=\cos \theta-z * \sin \theta \\
z^{\prime}=y * \sin \theta+z * \cos \theta x^{\prime}=x \\
z^{\prime}=z * \cos \theta-x * \sin \theta\end{array}$ & $S_{x}(\theta)=$ & $\left(\begin{array}{c}\cos \theta \\
0 \\
0\end{array}\right.$ & $\left.\begin{array}{cc}0 & 0 \\
\cos \theta & \sin \theta \\
-\sin \theta & \cos \theta\end{array}\right)$ \\
\hline 1 angmintit spint & $\begin{array}{l}x^{\prime}=z * \sin \theta+x * \cos \theta \\
y^{\prime}=y\end{array}$ & $S_{y}(\theta)=$ & $\left(\begin{array}{c}\cos \theta \\
0 \\
\sin \theta\end{array}\right.$ & $\left.\begin{array}{cc}0 & -\sin \theta \\
1 & 0 \\
0 & \cos \theta\end{array}\right)$ \\
\hline
\end{tabular}

$$
\begin{gathered}
S_{y z}=S_{y} * S_{z}=\left(\begin{array}{ccc}
\cos ^{2} \theta & -\sin \theta \cos \theta & -\sin \theta \\
\sin \theta & \cos \theta & 0 \\
\sin \theta \cos \theta & -\sin ^{2} \theta & \cos \theta
\end{array}\right) \\
S_{x z}=S_{x} * S_{z}=\left(\begin{array}{ccc}
\cos \theta & -\sin \theta & 0 \\
\sin \theta \cos \theta & \cos ^{2} \theta & \sin \theta \\
-\sin \theta & \sin \theta \cos \theta & \cos \theta
\end{array}\right)
\end{gathered}
$$

\section{Three-Dimensional Spin Revolution (3DSR) Scheme}

The designed scheme to discompose the information matrix $i_{\mathrm{m}}$ has the below entailed six phase processes.

Step 1: Choosing the alignment pair for spin.

Choosing an alignment pair, $a_{\mathrm{p}} \varepsilon\{x y, y z, x z\}$ based on which the revolution matrix is evaluated for $r_{\mathrm{m}}$.

$S_{a p}=S_{a} * S_{b}$

Here $a$ and $b \varepsilon x, y, z$ and $a \neq b$.

Step 2: Clustering the elements into trios.

Clustering the elements into $k$ trios $\left(e_{\mathrm{p}}, e_{\mathrm{q}}, e_{\mathrm{r}}\right)$ where $(p \neq q \neq r)$. The trios are clustered serially. Soon after clustering if one element remains the last, remaining last element will be clustered with the existing two elements. Likewise if two elements are prevailing after clustering, the last two elements are aggregated with the elements previous to them.

Step 3: Spin the trios around the alignment pairs $a_{\mathrm{p}}$ in a three-dimensional alignment for diverse angles of spin. Accomplish a three-dimensional revolution for each and every trio ' $n$ ' along the chosen alignment pairs $r_{\mathrm{m}}$ for acquiring the disconnected information set $d_{\text {is }}$.
$D_{\text {is }}=n\left(e_{\mathrm{p}}^{\prime}, e_{\mathrm{q}}^{\prime}, e_{\mathrm{r}}^{\prime}\right)=r_{\mathrm{m}} \times\left(e_{\mathrm{p}}, e_{\mathrm{q}}, e_{\mathrm{r}}\right)$

The values within the revolved information sets are the function of $\theta$ where $r_{\mathrm{m}}$ is the spin matrix about the $r_{\mathrm{m}}$ th alignment pair.

Step 4: Locating the angle of revolution ' $\theta$ '.

For the revolved information set, perform the below entailed as,

- For each and every trio, plan change of variation of the actual and disconnected information sets versus $\theta$ graphs.

- Originate three equations for each and every trios based on the limitations.

- Difference $\left(e_{\mathrm{p}}-e_{\mathrm{p}}^{\prime}\right) \geq l_{1}$, Difference $\left(e_{\mathrm{q}}-e_{\mathrm{q}}^{\prime}\right) \geq l_{2}$, Difference $\left(e_{\mathrm{r}}-e_{\mathrm{r}}^{\prime}\right) \geq l_{3}$ here $l_{1}>0, l_{2}>0$ and $l_{3}>0$.

- Locate the extent for ' $\theta$ ' which fulfils the safety related fixed values for $l_{1}, l_{2}$ and $l_{3}$. These extents are termed as safety extent.

- Based on the connection of safety extent of all the trios acquired from the preceding steps, select an arbitrary real value $\varphi$ as $\theta$.

Step 5: Disconnect the information by revolving at an angle $\theta$.

Measure the spinning trios at an angle of $\theta$ located from step 4 .

i.e. $D_{i s}=n\left(e_{p}^{\prime}, e_{q}^{\prime}, e_{r}^{\prime}\right)=r_{m} \times\left(e_{p}, e_{q}, e_{r}\right)$

Step 6: Selection of information to be liberated.

For each and every disconnected information set $S_{x y}, S_{y z}$ and $S_{x z}$, measure the differences. The information $i_{\mathrm{m}}$ with the utmost value of differences is chosen as the concluding disconnected information $i_{\mathrm{m}}$. 


\section{Three-Dimensional Spin Revolution (3DSR) Algorithm}

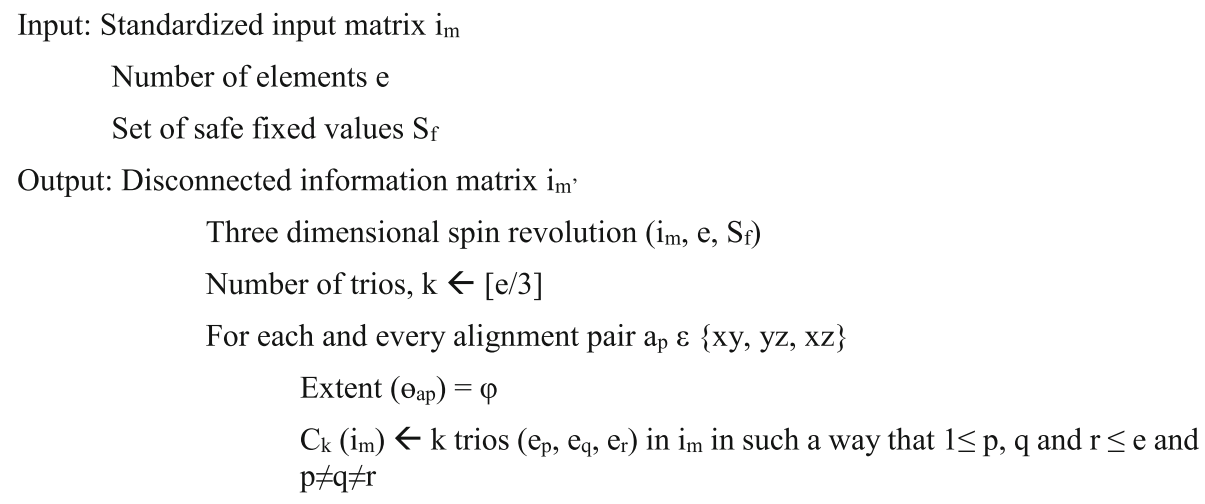

For diverse values of $\theta$

For each and every $c_{\mathrm{k}}$ in $\mathrm{C}_{\mathrm{k}}\left(\mathrm{i}_{\mathrm{m}}\right)$

Evaluate $D_{i s}=n\left(e_{p}^{\prime}, e_{q}^{\prime}, e_{r}^{\prime}\right)=r_{m} \times\left(e_{p}, e_{q}, e_{r}\right)$ where $\mathrm{n}$ is the function of $\mathrm{\theta}$

End for

Evaluate the differences

End for

Plot the differences versus $\Theta$ graph

Evaluate the safety extent of $\Theta_{\mathrm{k}}$, extent $\left\{\theta_{\mathrm{k}}\right\}$ in a way that Difference $\left(e_{p}-e_{p}\right) \geq 1_{1}$, Difference $\left(e_{q}-e^{\prime}{ }_{q}\right) \geq 1_{2}$, Difference $\left(e_{r}\right.$ $\left.-\mathrm{e}_{\mathrm{r}}^{\prime}\right) \geq 1_{3}$ where $\mathrm{l}_{\mathrm{n}} \varepsilon \mathrm{S}_{\mathrm{f}}$.

Extent $\left\{\theta_{\mathrm{k}}\right\} \leftarrow$ extent $\left\{\boldsymbol{\theta}_{\mathrm{ap}}\right\} \wedge$ extent $\left\{\boldsymbol{\theta}_{\mathrm{k}}\right\}$

$\Phi_{\text {ap }} \leftarrow$ revolution angle such that $\Phi_{\text {ap }} \varepsilon$ 䭪

Estimate $D_{i s}=n\left(e_{p}^{\prime}, e_{q}^{\prime}, e_{r}^{\prime}\right)=r_{m} \times\left(e_{p}, e_{q}, e_{r}\right)$

Evaluate $\mathrm{d}_{\mathrm{is}}=$ difference of $\mathrm{d}_{\mathrm{is} \varphi}$

End for

$\mathrm{i}_{\mathrm{m}}, \leftarrow \mathrm{d}_{\mathrm{is} \varphi}$ with utmost $\mathrm{n}_{\mathrm{ap}}$

end

\section{Complexity Analysis}

Let ' $O$ ' represent the number of entities and $a_{n}$ denote the number of elements. The execution time of the designed three-dimensional spin revolution scheme is $O\left(m^{*} n\right)$ (Table 3).

Table 3 Variables used in 3DSR

\begin{tabular}{ll}
\hline Variables & Corresponding meanings \\
\hline$i_{\mathrm{m}}$ & Information matrix \\
$e$ & Number of elements \\
$S_{\mathrm{f}}$ & Set of safe fixed values \\
$\theta_{\mathrm{ap}}$ & Extent \\
$n$ & Function of $\theta$ \\
$\Phi_{\mathrm{ap}}$ & Revolution angle \\
$d_{\text {is }}$ & Difference of $d_{\mathrm{is} \varphi}$ \\
\hline
\end{tabular}

\section{Performance Analysis}

The MATLAB scripts are employed for creating difference angle graphs and to disconnect the information set. The bank marketing information set from the UCI database is employed to authenticate the precision and effectiveness of the three-dimensional spin revolution schemes. The bank marketing information sets hold the description of about 50,200 calls employed for straightforward marketing promotions of a Portuguese management institution. It comprised nearly 18 statistical input elements among which eight are arithmetic and nine are unqualified. The result variable is a binary class element with values 1 or 0 . Only arithmetic is a binary class element which is considered to be of concern in terms of age, equalization, day, length, promotion and preceding days. The classified elements are safeguarded employing normalized encoding-based scheme, namely DES. The information set is standardized 


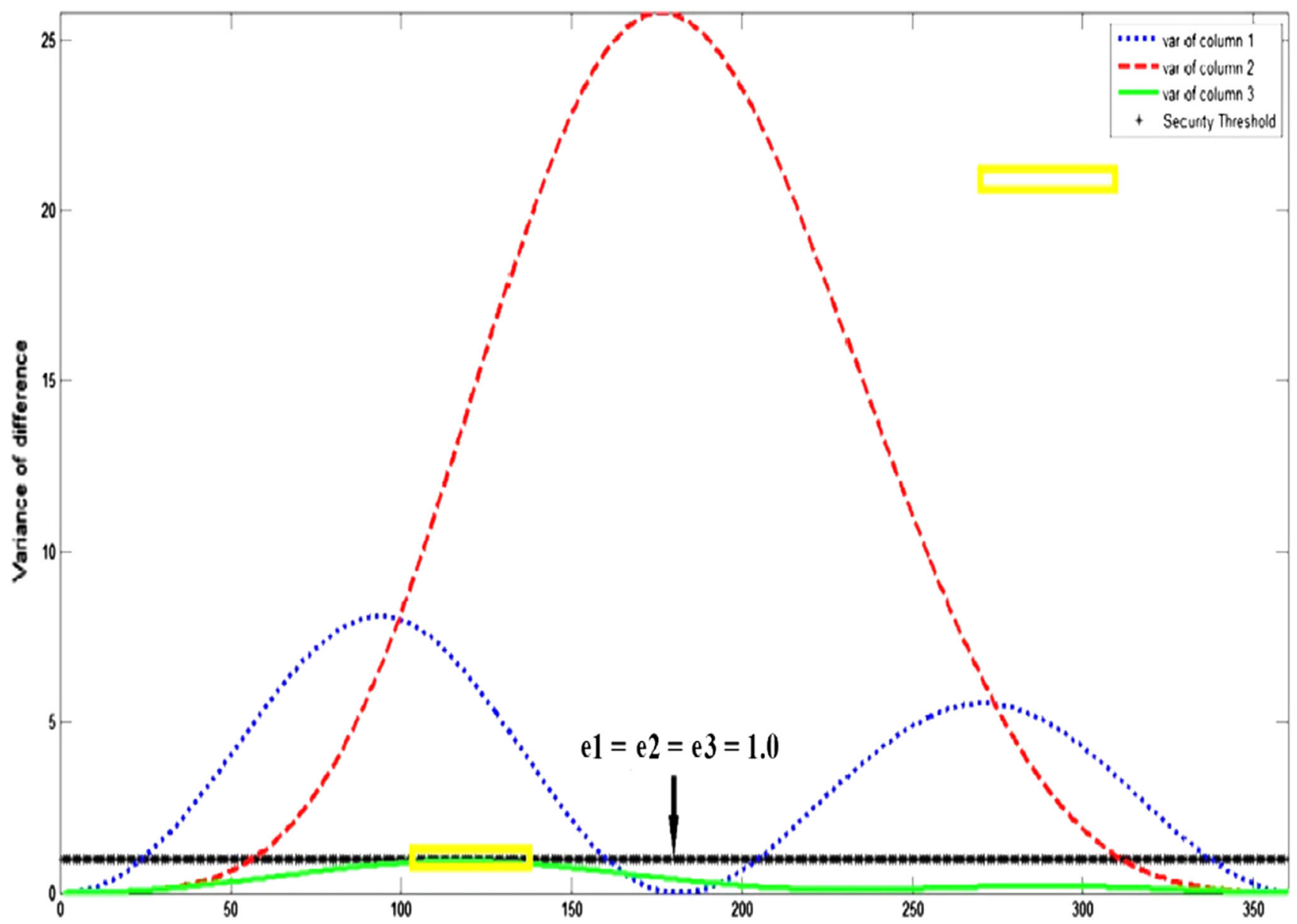

Fig. 3 Differences versus dimension for $t_{1}$

using MIN_MAX and modifying the element values into the extent $[0,5]$. The eight arithmetic element values are clustered into trios as, $a_{n 1}=\left[\right.$ element $_{1}$, element ${ }_{2}$, element $\left._{3}\right], \quad a_{n 2}=\left[\right.$ element $_{4}$, element 5 , element 6 and $a_{n 3}$ $=\left[\right.$ element $_{5}$, element 6 , element 7 .

Each and every trio is allocated a safety fixed values as,

$S_{f 1}=(1,1,1), \quad S_{f 2}=(0.5,0.5,0.5), \quad S_{f 3}=(0.5)$

Each and every trio acquired is revolved about an alignment pair ( $x y, y z$ and $x z$ ) with the intention to escalate the volume of disconnection to objects without bothering the spatial distances. For each and every revolution, the relative spin matrix is estimated as entailed in Fig. 3. For diverse values of $\theta$ estimate, the revolved trio $n^{\prime}=n * s_{a p}$ and plots a graph among the angle $\theta$ and variety of differences.

It offers the extent of $\theta$ such a way that minimal safety fixed value pre-requisites for each and every element is fulfilled based on $\theta$. The differences versus angle graphs for three trios revolved along the $y z$ alignment. The extent of safety for $\theta$ for $a_{n 1}$ is $110-140, a_{n 2}$ is the extent $45-150$ and $230-340$ and $a_{n 3}$ is $50-350$. From the connection of these dimensions of $\theta$ an angle $\theta$ is selected by 130 angles, which offers a covered extent of the exploited difference between the actual and the disconnected information. Replacing the values of $\varphi$ in $n$ ' for each and every trio, the distorted repositories $d_{\text {is }}$ are acquired. For the bank repository, the difference $p$ after the revolution related to the diverse alignment pairs is depicted in Table 2.

In Fig. 4, we are taking the $e 1, e 2$ and $e 3$ values as 0.5 . In this figure, we got two safety extent and we are considering columns 4, 5 and 6 . The blue colour lines indicate the column 4 , the red colour line indicates the column 5 , and the green colour indicates column 6 . Here we involved three spinning and in the column 6 got the maximum variance of difference.

In Fig. 5, we involved only the variance of column 7 and we got the e value 0.4 . Also we got the maximum variance difference and compared to other two, here we got the most safety extent.

In Fig. 4, we are comparing the output of two-dimensional revolution technique (2DRT) with three-dimensional revolution technique (3DRT). When comparing these two methods, the variance of three-dimensional spinning is five times greater than of two-dimensional spinning. 
J. Inst. Eng. India Ser. B (April 2021) 102(2):387-402

399

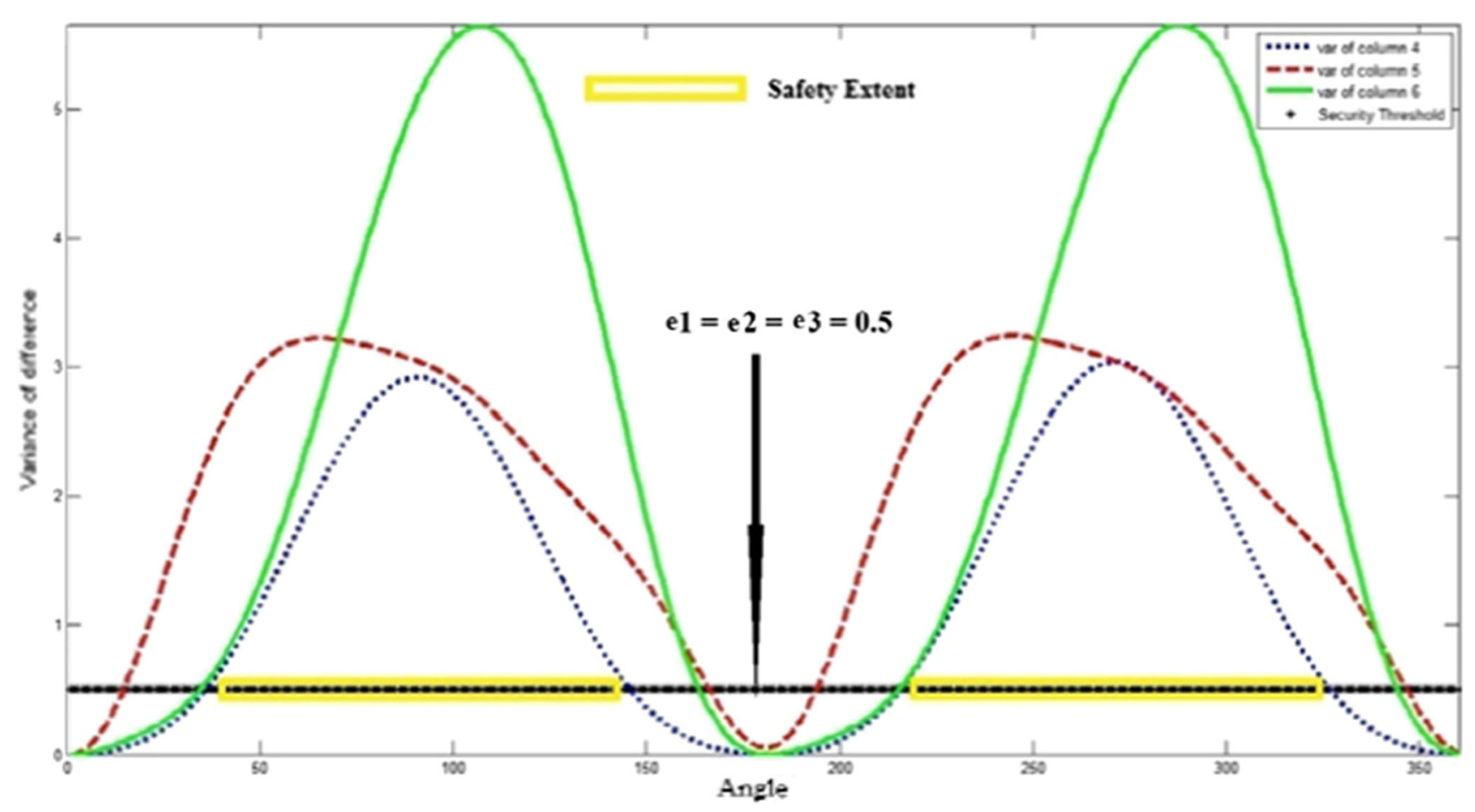

Fig. 4 Differences versus dimension for $t_{2}$

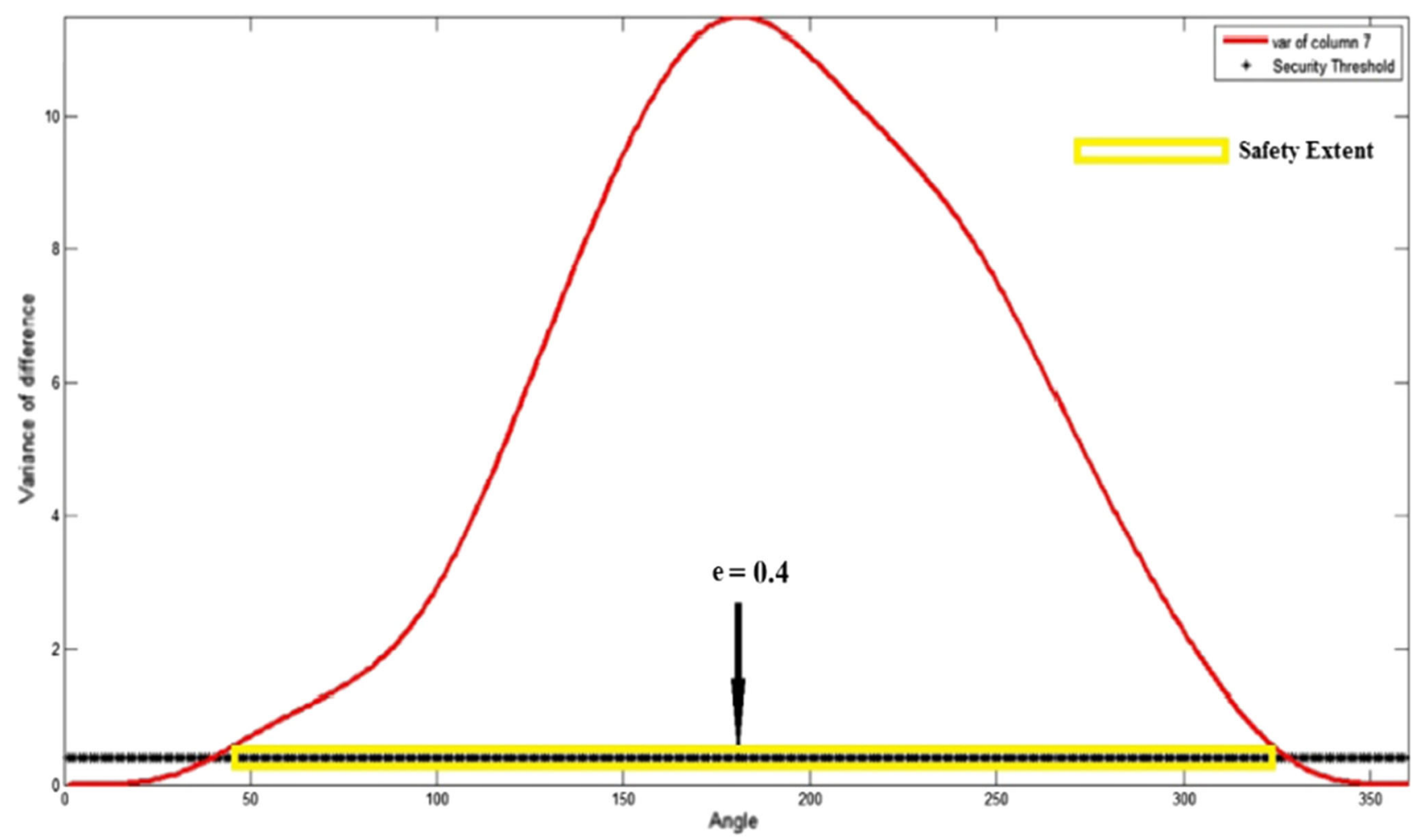

Fig. 5 Differences versus dimension for $t_{3}$

Springer 
Table 4 Evaluation of difference pair alignment of axes-bank information set

\begin{tabular}{ll}
\hline Axes of revolution & Differences \\
\hline$y-z$ & $2.9876 * \mathrm{e}+006$ \\
$x-z$ & $4.0001 * \mathrm{e}+005$ \\
$x-y$ & $1.066 * \mathrm{e}+003$ \\
\hline
\end{tabular}

From Table 4, it is profound that the bank information set $y z$ pair offers the improved confidentiality estimates as the difference related to $y z$ pair is the maximum. Hence the disconnected information set related to $y z$ pair is regarded as the last disconnected information $i_{\mathrm{m}}$.

\section{Results and Discussion}

In order to authenticate the precision and effectiveness of the designed disconnected schemes, the outcomes of the experiment performed are based on two features such as confidentiality management and precise extraction.

\section{Capability in Confidentiality Safeguarding}

The confidentiality safeguarding abilities are calculated based on the difference parameter. These differences are an arithmetic value employed to represent the manner in a wide manner where the clusters differ. The differences are employed to calculate the differences among the actual information and disconnected information. The differences are measured based on the equation.

$\operatorname{Difference}\left(n_{1}, n_{2}, \ldots n_{n}\right)=\frac{1}{e} \sum_{i=1}^{n}\left(\left|e_{i}-\vartheta\right|\right)^{2}$

Here $\vartheta$ represents the differences.

The difference-based confidentiality parameter for the disconnected information set $i_{\mathrm{m}}$, is entailed as,

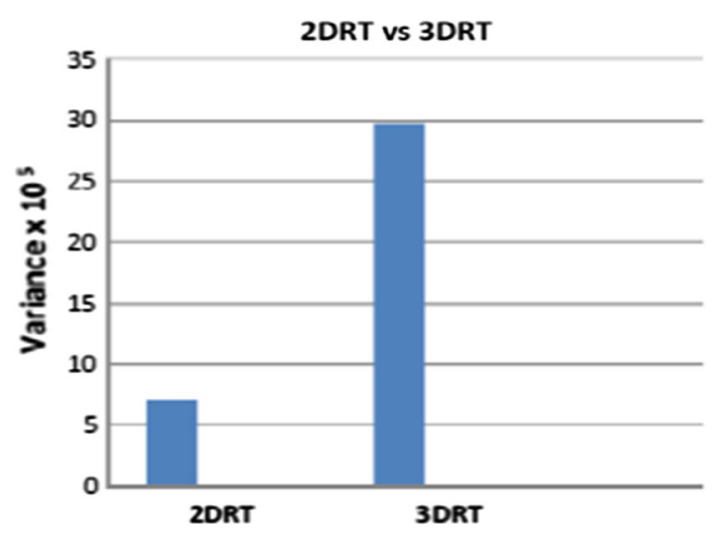

Fig. 6 Evaluation of abilities in safeguarding confidentiality $p=\operatorname{difference}\left(\frac{\text { Difference }\left(c_{\mathrm{a}}-c_{\mathrm{a}}\right)}{\operatorname{Difference}\left(c_{\mathrm{a}}\right)}\right)$

Here the $c_{\mathrm{a}}$ represents the actual information matrix and $c_{\mathrm{a}}$ denotes the relative column of the disconnected information matrix.

The confidentiality safeguarded abilities of the designed three-dimensional spin revolution evaluated against the two-dimensional spin revolution are depicted in Fig. 6. It is evident that the three-dimensional spin revolution is five times better than the two-dimensional spin revolution.

\section{Precision in Extracting Information}

For estimating the outcomes of the disconnected information in information extraction, four categorization prototypes such as simple Bayes categorizer, decision table, $k$-adjacency categorizer and decision tree categorizers are chosen. The simulation tool is employed to acquire the outcomes of information extraction for these categorization prototypes. The chosen evaluation is the categorized precision which is entailed as the proportion of precisely categorized illustrations to the overall number of illustrations. A tenfold crossauthentication is employed for testing the categorizers. A two-dimensional disconnected, three-dimensional disconnected and actual information sets are extracted employing the four categorization-based schemes, and the outcomes are depicted in Fig. 7. It is evident that the precision in categorization on the disconnected information is nearly similar to that of actual information.

\section{Conclusion}

The intention is to design a fresh confidentiality safeguarded information modification scheme that could be employed with diverse varieties of information extraction prototypes. The three-dimensional spin revolution

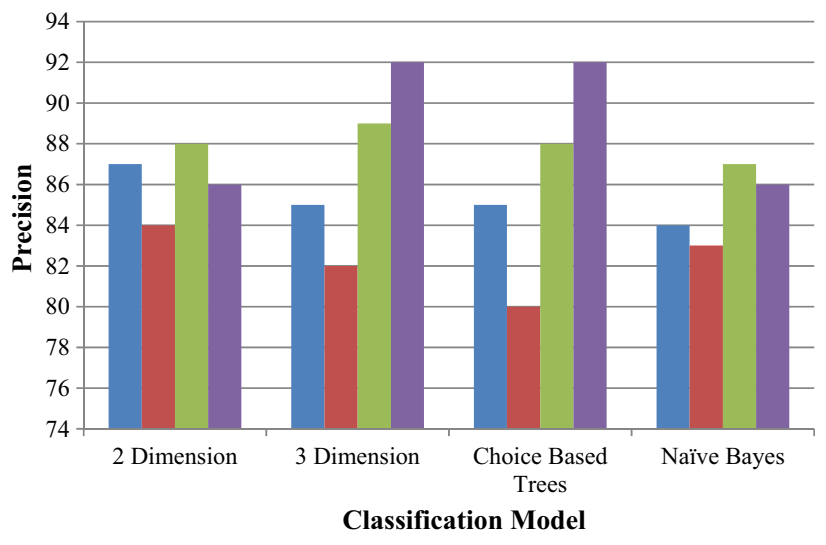

Fig. 7 Evaluating the precision of data mining 
scheme designed for the information is revolved twice along two diverse alignments. It improves the differences making the information tougher to threats. Furthermore, the disquiets of information are not bothering much the abilities in information extraction of the Euclidean distances.

The results of simulation reveal that the precision in information extraction of the actual and disconnected information is closely identical and has increased differences which depict its utmost abilities in safeguarding confidentialities. The intention is to design a fresh confidentiality safeguarded information modification scheme that could be employed with diverse varieties of information extraction prototypes. The three-dimensional spin revolution scheme designed for the information is revolved twice along two diverse alignments. The forthcoming entails the conclusions of all the proposed schemes along with possible extensions.

\section{References}

1. A. Rahman, M.N.A. Khan, An assessment of data mining based CRM techniques for enhancing profitability. Int. J. Educ. Manag. Eng. 2, 30-40 (2017)

2. A. Sachan, D. Roy, P.V. Arun, An analysis of privacy preservation techniques in data mining, in Proceedings of the International Conference on Advances in Computing and Information Technology (2017), pp. 119-128

3. A.-E.-E.A. Hussein, N. Hamza, H.A. Hefny, Attacks on anonymization based privacy preserving: a survey for data mining and data publishing. J. Inf. Technol. 4, 101-112 (2013)

4. A.Y. Noaman, F. Nadeem, A.H.M. Ragab, A. Jamjoom, N. AlAbdullah, M. Nasir, A.G. Ali, Improving Prediction Accuracy of 'Central Line-Associated Blood Stream Infections' Using Data Mining Models. BioMed Res. Inter. 2017, 1-12 (2017)

5. A. Sheshasaayee, L. Lageshari, An efficiency analysis on the TPA clustering methods for intelligent customer segmentation, in Proceedings of the International Conference on Innovative Mechanisms for Industry Applications, IEEE (2017)

6. A. Patel, P. Shreya, K. Amin, A survey on heuristic based approach for privacy preserving in data mining. Int. J. Sci. Res. Comput. Sci. Eng. 5(2), 21-25 (2017)

7. M. Antony Sheela, K. Vijayalakshmi, Partition based perturbation for privacy preserving distributed data mining. Int. J. Cybern. Inf. Technol. 17(2), 44-55 (2017)

8. B.S. Kumar, S. Karthik, V.P. Arunachalam, Upkeeping secrecy in information extraction using ' $\mathrm{k}$ ' division graph based postulates. Clust. Comput. 22(S1), 57-63 (2018)

9. C. Sharma, K. Garg, A novel filtering based scheme for privacy preserving data mining. Int. Res. J. Eng. Technol. 4(8), 2005-2011 (2017)

10. Kantarcioglu and Murat, A survey of privacy-preserving methods across horizontally partitioned data, in Privacy-Preserving Data Mining and Advances in Database Systems, vol. 34 (Springer, 2008), pp. 313-335

11. M. Panda, A.E. Hassanien, A. Abraham, Hybrid data mining approach for image segmentation based classification. J. Rough Sets Data Anal. 2(3), 65-81 (2016)
12. S. Yousef, S. Karunan, Satellite image mining in real time data analytical architecture. IOSR J. Comput. Eng. 1(11), 49-54 (2017)

13. S.S. Liu, J. Chan, Using data mining to segment healthcare markets from patients preference perspectives. Int. J. Healthc. Qual. Assur. 22(2), 117-134 (2009)

14. P. Saranya, S. Kumar, A survey on feature selection of cancer disease using data mining techniques. Int. J. Comput. Sci. Mob. Comput. 5(5), 713-719 (2016)

15. S. Lohiya, P. Ramayi, A. Pillai, S. Veturi, Privacy feedback system using data mining and outlier detection algorithm. Int. J. Innov. Res. Comput. Commun. Eng. 5(3), 3232-3240 (2017)

16. S. Gupta, H. Bhadkambar, Survey paper on privacy preserving data mining techniques. Int. J. Eng. Sci. Res. Technol. 6(10), 164-168 (2017)

17. S. Mogtaba, E. Kambal, Association rule hiding for privacy preserving data mining, in Proceedings of the International Conference on Advances in Data Mining, Applications and Theoretical Aspects (2016), pp. 320-333

18. S. Shinde, V. Singan, S. Asati, Data leakage detection in data mining system. Int. J. Res. Emerg. Sci. Technol. Special-Issue 1, 105-108 (2017)

19. T.P.K. Asiabi, R. Tavoli, A review of different data mining techniques in customer segmentation. J. Adv. Comput. Res. 6(3), 51-63 (2015)

20. A.V. Kelarev, X. Yi, H. Cui, L. Rylands, H.F. Jelink, A survey of state of the art methods for securing medical databases. J. Med. Sci. 5(1), 1-22 (2017)

21. A.W. Putri, L. Hira, Hybrid transformations in privacy preserving data mining, in Proceedings of the International Conference on Data and Software Engineering, IEEE (2017)

22. R. Kaur, K. Kaur, Data mining on customer segmentation: a review. Int. J. Adv. Res. Comput. Sci. 8(5), 261-265 (2017)

23. V.S. Verykios, E. Bertino, I.N. Fovino, L.P. Provenza, Y. Saygin, Y. Theodoridis, State of the art in privacy preserving data mining. Newsl. SIGMOID 33(1), 50-57 (2004)

24. J. Vinothkumar, V. Santhi, An extensive survey of privacy preserving data mining approaches for medical datasets. Int. J. Pharm. Technol. 8(4), 4979-4988 (2016)

25. W. Cheng, M. Zhao, N. Xiong, K.T. Chui, Non-convex sparse and low-rank based robust subspace segmentation for data mining. Sens. J. 17, 1633 (2017)

26. Z. Ge, Z. Song, S.X. Ding, B. Huang, Data mining and analytics in the process industry: the role of machine learning. IEEE Trans. Data Min. 5, 20590-20616 (2017)

27. R. Geetha Ramani, S. Sugirtharani, B. Lakshmi, Automation detection of glaucoma in retinal fundus images through image processing and data mining techniques. Int. J. Comput. Appl. 166(8), 38-43 (2017)

28. H. Roshan, M. Afsharinezhad, The new approach in market segmentation by using RFM model. J. Appl. Res. Ind. Eng. 4(4), 259-267 (2017)

29. Jason Pridmore and Lalu Elias and Hamalainen, Market segmentation in action: marketing and 'yet to be installed' role of big and social media data. SSOAR J. 42, 103-122 (2017)

30. J. Li, J. Wei, W. Liu, X. Hu, PMDP: a framework for preserving multiparty data privacy in cloud computing. J. Secur. Commun. Netw. (2017)

31. T. Dinkar, A. Patel, K.R. Amin, Preserving the Sensitive Information Using Heuristic Based Approach (IEEE, New York, 2016).

32. A. Tripathy, M. Pradhan, A novel framework for preserving privacy of data using correlation analysis, in Proceedings of International Conference on Advances in Computing Communications and Informatics (2012) 
33. C. Alexandre, P. Bocsanean, J. Mangana, Marketing behaviours analysis in a mobile wallet solution using data mining. Int. J. Comput. Appl. 150(11), 42-53 (2017)

34. M. Chalapathi Rao, A. Kiran Kumar, Challenges arise of privacy preserving big data mining techniques. Int. Res. J. Eng. Technol. 4(5), 1-8 (2017)

35. E. Stankevicius, K. Kundaeliene, Theoretical approach to tax1036 payers segmentation. J. Contemp. Issues Bus. Manag. Educ. ISSN 2029-7963. ISBN 9786094760129, pp: 127-135 (2017)

36. F. Farazzmanesh, Analysis of business customers value network using data mining techniques. Int. J. Comput. Appl. 121(10), 1-6 (2017)

37. P. Gayathiri, B. Poorna, Association rule hiding for privacy preserving data mining: a survey on algorithmic classifications. Int. J. Appl. Eng. Res. 12(23), 13917-13926 (2017)

38. H. Chabanne, A. de Wargny, J. Milgrun, Privacy preserving classification on deep neural network. Int. J. Comput. Appl. 120(10), 1-7 (2016)

39. H. Divecha, S. Mehta, Privacy preserving based on geometric transformation using data perturbation technique. Int. J. Softw. Hardw. Res. Eng. 2(5), 1-9 (2014)

40. J. Schutte, A. Van Der Merwe, F. Reyneke, Using data analytics and data mining methods to determine a high net worth individuals electronic banking behavior. J. Internet Bank. Commer. 22(3), 1-39 (2017)
41. J.C.-W. Lin, T.P. Hong, P.F. Viger, Q. Liu, J.W. Wong, J. Zhan, Efficient hiding of confidential high utility item sets with minimal side effects. J. Exp. Theor. Artif. Intell. 29(6), 1225-1245 (2017)

42. Karim Abouelmehdi, Abderrahim Beni - Hessane and Hayat Khaloufi, 2018, 'Big Healthcare Data: Preserving Security and Privacy', Journal of Big Data, Vol. 5, No. 1.

43. Mohammad Hasan Aghdaie, Sep. 2017, 'Data Mining Group Decision Making With Fuzzy AHP: An Application in Supplier Evaluation and Segmentation', ResearchGate Publication.

44. N. Rattanamethawong, S. Sinthupinyo, A. Chandrachai, An innovation model of alumni relationship management: alumni segmentation analysis. J. Soc. Sci. 39(1) 1-11. (2017)

45. P.D. Bagul, Waghmare, A survey of data mining techniques for improvement of prediction analysis. Int. J. Innov. Res. Comput. Commun. Eng. 5(3), 1-6 (2017)

46. P.S. Kade, N.M. Dhande, A paper on web data segmentation for terrorism detection using named entity recognition technique. Int. Res. J. Eng. Technol. 4(1), 217-222 (2017)

47. N. Rajesh, K. Sujatha, A. Lawrence, Survey on privacy preserving data mining techniques using recent algorithms. Int. J. Comput. Appl. 133(7), 30-33 (2016)

Publisher's Note Springer Nature remains neutral with regard to jurisdictional claims in published maps and institutional affiliations. 